\title{
The Value of Intravoxel Incoherent Motion (IVIM) Magnetic Resonance Imaging in the Evaluation of the Osteosarcoma Response to Preoperative Neoadjuvant Chemotherapy in Teens
}

\author{
Houdong Zuo \\ Sichuan Key Laboratory of Medical Imaging; Department of Radiology, Affiliated Hospital of North Sichuan Medical College, \\ Nanchong 637000, China
}

Correspondence should be addressed to Houdong Zuo; zuohoud@163.com

Received 5 March 2019; Revised 17 April 2019; Accepted 30 April 2019; Published 9 May 2019

Academic Editor: Yi Wang

Copyright (c) 2019 Houdong Zuo. This is an open access article distributed under the Creative Commons Attribution License, which permits unrestricted use, distribution, and reproduction in any medium, provided the original work is properly cited.

\begin{abstract}
Purpose. To investigate the value of intravoxel incoherent motion (IVIM) magnetic resonance imaging in the evaluation of the osteosarcoma (OS) response to preoperative neoadjuvant chemotherapy (NAC) in teens. Methods. Eighteen consecutive osteosarcoma patients (12 males and 6 females) diagnosed by histopathology were enrolled. All enrolled patients received NAC and underwent MRI examinations before and after 2 cycles of NAC. The volume (V), apparent diffusion coefficient (ADC), (slow) diffusion coefficient (D), pseudo-perfusion (fast) diffusion coefficient ( $\mathrm{D}^{*}$ or $\mathrm{D}$ star), and perfusion fractions $(f)$ of the OS before and after NAC were measured and analysed. The sensitivity and the specificity of the ADC, $\mathrm{D}, \mathrm{D}^{*}$ and $f$ values and the correlation between changes in volume $\left(\Delta_{\mathrm{V}}\right)$ and the IVIM-derived parameters $\left(\Delta_{\mathrm{ADC}}, \Delta_{\mathrm{D}}, \Delta_{\mathrm{D} *}\right.$ and $\left.\Delta_{f}\right)$ were also calculated and analysed. Paired Student's t-tests, receiver operating characteristic (ROC) curves, and Spearman correlation analyses were used. Results. The mean volume of the OS after NAC decreased significantly $\left(61.55 \pm 9.3 \mathrm{~cm}^{3}\right.$ vs $\left.40.26 \pm 4.1 \mathrm{~cm}^{3}\right)(\mathrm{P}<0.05)$. After NAC, the ADC $\left(1.21 \pm 0.09 \times 10^{-3} \mathrm{~mm}^{2} / \mathrm{s}\right.$ vs $\left.1.73 \pm 0.11 \times 10^{-3} \mathrm{~mm}^{2} / \mathrm{s}\right)$ and $\mathrm{D}\left(1.14 \pm 0.09 \times 10^{-3} \mathrm{~mm}^{2} / \mathrm{s}\right.$ vs $\left.1.63 \pm 0.1 \times 10^{-3} \mathrm{~mm}^{2} / \mathrm{s}\right)$ values increased and $f$ $(28.58 \pm 1.37 \%$ vs $20.93 \pm 1.16 \%)$ values decreased significantly $(\mathrm{P}<0.05)$, while $\mathrm{D}^{*}\left(9.17 \pm 0.98 \times 10^{-3} \mathrm{~mm}^{2} / \mathrm{s}\right.$ vs $\left.8.94 \pm 0.13 \times 10^{-3} \mathrm{~mm} / \mathrm{s}\right)$ values changed slightly $(\mathrm{P}>0.05)$. The AUC values of $\mathrm{ADC}(0.938)$ and $\mathrm{D}(0.943)$ were higher than those of $\mathrm{D}^{*}(0.574)$ and $f(0.765)$. The sensitivity and specificity of $\operatorname{ADC}(0.778,1)$ and $\mathrm{D}(0.944,0.833)$ values were superior to those of $\mathrm{D}^{*}(0.278,0.722)$ and $f(0.5$, $0.944)$ values. No significant correlations were found between the $\Delta_{\mathrm{V}}$ and $\Delta_{\mathrm{ADC}}, \Delta_{\mathrm{D}}, \Delta_{\mathrm{D} *}$ and $\Delta_{f}$ values $(\mathrm{P}>0.05)$. Conclusion. IVIM-derived parameters $\mathrm{ADC}$ and $\mathrm{D}$ have the potential to be imaging biomarkers for evaluation of the therapeutic response to NAC in OS patients.
\end{abstract}

\section{Introduction}

Osteosarcoma (OS) is a common cancer of the musculoskeletal system in teens and is a leading cause of cancer death and disability worldwide [1]. The most involved sites are the distal femur, the proximal humerus, the proximal tibia, and the metaphysis [2]. The age of peak incidence rate ranges from 14 to 26 years old, especially 14 to 19 years old, and this constitutes a separate specific subgroup who suffer osteosarcoma [3]. The clinical manifestations of OS include local persistent pain, palpable lumps, swelling, dysfunction, and pathological fractures [4]. Osteosarcoma has a poor prognosis because of its highly malignant biological characteristics and metastatic tendencies $[5,6]$. The five-year relative survival rates (\%) for childhood and adolescent OS in the United States from 2007 to 2013 ranged from $69.8 \%$ to $69.5 \%$ [1]. The current therapeutic methods adopted for OS are surgical resection and clinical chemotherapy, especially neoadjuvant chemotherapy (NAC) [7-9]. The purpose of osteosarcoma neoadjuvant chemotherapy is to reduce the primary tumour size, eliminate (micro-)metastatic deposits, maximize limb function retention and minimize patient suffering, trauma and treatment-related adverse outcomes [10, 11]. NAC has been proven to be ineffective in approximately half of OS patients [12]. Therefore, reliable early imaging predictors are vital to early evaluation of the OS treatment response of NAC to optimize the therapeutic regimen individually and reduce ineffectively toxic therapy. [12]. 
There has been growing interest in utilizing functional imaging techniques to predict early therapeutic efficacy. Conventional magnetic resonance imaging (MRI) can be used to evaluate the tumors and metastasis in pretherapeutic patients, but reevaluating after therapy is also challenging [13]. In addition, diagnostic assessment by using conventional MRI may be hampered by difficulties in distinguishing residual tumor from NAC-induced fibrosis, and the edema can potentially lead to being over-evaluated [14]. Diffusionweighted imaging (DWI), which is based on the Brownian motion of water molecules and can reflect the thermal motion of water molecules at the cellular levels [15], may be helpful in differentiation and thus improve the early prediction of response after NAC [16]. Therapy-induced cellular necrosis gives rise to increasing water diffusivity. The apparent diffusion coefficient (ADC) is currently used to reflect the magnitude of diffusion and can be calculated with a monoexponential function. But along with pure molecular diffusion, microcirculation within capillary networks also contributes to the ADC value [16]. Here, a functional MRI technique, intravoxel incoherent motion (IVIM), was introduced to separate microcapillary perfusion from DWI data with multiple $b$ values in biexponential model and has been widely investigated in the assessment of therapeutic responses before and after chemotherapy in many malignant tumours [17, 18]. Le Bihan et al. [19] proposed the IVIM dual exponential imaging mode with multiple $b$ values to depict perfusion and diffusion effects in tissues [20]. Another advantage of IVIM is that the derived parameters, including the apparent diffusion coefficient (ADC), (slow) diffusion coefficient (D), pseudo-perfusion (fast) diffusion coefficient $(\mathrm{D} *)$, and perfusion fractions $(f)$, can be calculated without contrast injection $[19,21]$. ADC is sensitive to water molecules in the tissues and can reflect tissue diffusion and perfusion [22]; the $\mathrm{D}$ value is generally considered to be the pure diffusion coefficient, reflecting extracellular and extravascular tissue water molecular motion $[23,24]$. The $f$ and $\mathrm{D} *$ values have the advantage of reflecting tumour angiogenesis and correlating with microvessel density (MVD) scores in tumours [24, 25]. Because of the great potential in the tumour evaluation and grading, IVIM is used widely to assess the NAC therapeutic responses in various malignant tumours [26-28]. To date, few studies have reported application of the IVIM technique in OS and focus on the evaluation of the OS response to NAC. Thus, the aim of this study was to investigate the value of the IVIM in evaluating the OS therapeutic response to NAC and determine whether the IVIM-derived parameters could serve as imaging biomarkers in OS patients with NAC.

\section{Materials and Methods}

2.1. Patients. This study was approved by the institutional review board of our hospital. Written informed consent was obtained from all patients before the study, and the study was performed according to the ethical guidelines of our institutional clinical research committee. Twelve male (mean age, $17.25 \pm 1.42$, range, $15-20$ years) and 6 female (mean age, $17.5 \pm 1.05$, range, $16-19$ years $)$ consecutive patients $(n=18$, mean age, $17.33 \pm 1.28$, range, $15-20$ years) with osteosarcoma diagnosed by histopathology were enrolled in this study between May 2016 and September 2018. To guarantee the accuracy of the results, the OS located in the distal femoral metaphysis were selected. All patients enrolled who received NAC and underwent knee MRI examinations in this study met the following criteria: (1) all patients had completed NAC and surgery; (2) the first MRI was performed before the initiation of NAC; (3) the second MRI was performed after the completion of NAC; and (4) the NAC course was 2 cycles ( 4 weeks). The NAC regimens consisted of cisplatin (100$\left.120 \mathrm{~g} / \mathrm{m}^{2}\right)$ and doxorubicin $\left(60 \mathrm{~g} / \mathrm{m}^{2}\right)$. Patients were given folic acid during chemotherapy. The treatment response and physical situation were observed closely and recorded. The patients in this study did not receive radiotherapy.

2.2. Treatment Response Analysis. Therapeutic response was determined by the final tumor size on MRI 2 cycles after NAC. The responses could be classified clinically into four types based on RECIST: (1) Complete response (CR), no residual tumor tissues on T2WI; (2) partial response (PR), the maximum diameter of the tumor less than $70 \%$ of the original size; (3) progressive disease (PD), at least a $20 \%$ increase in the sum of longest diameter of the tumor; (4) stable disease, neither sufficient shrinkage to be as PR nor sufficient increase to be as PD. The maximum diameter was recorded before treatment as the reference [29].

2.3. MR Imaging. All patients underwent whole-body $3.0 \mathrm{~T}$ MR scans (Discovery MR750; GE Healthcare, MI) using a special knee coil. Conventional MRI sequences included axial spin-echo T1-weighted images (T1WI) (TR/TE, 512/12.4 ms), axial, coronal, and sagittal fast spin-echo T2-weighted images (T2WI) with or without fat suppression (2500/71 ms). Axial diffusion weighted imaging (DWI) was performed using single-shot spin-echo echo-planar imaging sequence $(3225 / 69.2 \mathrm{~ms})$ with $256 \times 256$ matrix, slice thickness: $5 \mathrm{~mm}$, slice gap: $1 \mathrm{~mm}$, field of view (FOV): 150-200, EPI factor of 5, and $b$-values of $0,50,150,200,400,800,1000$ and $1200 \mathrm{~s} / \mathrm{mm}^{2}$; the total scan time was $4 \mathrm{~min}$ and 24 seconds.

For the dynamic contrast-enhanced MRI (DCE-MRI) sequence, the transverse $3 \mathrm{D}$ fast spoiled gradient recalled echo (FSPGR) sequence with fat saturation was scanned after intravenous administration of $0.1 \mathrm{mmol} / \mathrm{kg}$ Gd-DTPA (Magnevist, Germany) at $2 \mathrm{ml} / \mathrm{s}$ (with a flush of $20 \mathrm{~mL}$ saline solution) via a high-pressure injector within 15 seconds.

2.4. MRI Data and Image Analysis. The conventional sequences (T1WI, T2WI) and DCE are used to be the auxiliary methods to observe and measure the OS, including the signal intensity (SI), size and volume (V) changes before and after NAC. The volume was calculated with the equation $\mathrm{V}=\mathrm{L} \times \mathrm{W} \times \mathrm{W} / 2$ (V: volume; L: length; W: width) [30]. The volume changes of the OS $(\Delta \mathrm{V})$ were measured and calculated $\left(\Delta_{\mathrm{V}}=\left|\mathrm{V}_{\text {before } \mathrm{NAC}}-\mathrm{V}_{\text {after NAC }}\right|\right)$.

ADC values were calculated using a monoexponential model with all the $b$ values [19]: 
TABLE 1: IVIM imaging parameter mean values before and after NAC.

\begin{tabular}{|c|c|c|c|c|c|c|}
\hline & Before NAC & After NAC & Sensitivity & Specificity & $\mathrm{P}$ value & Cutoff values \\
\hline $\begin{array}{l}\mathrm{ADC}_{\text {mean }} \\
\left(\times 10^{-3} \mathrm{~mm}^{2} / \mathrm{s}\right)\end{array}$ & $1.21 \pm 0.09$ & $1.73 \pm 0.11$ & 0.778 & 1 & $<0.001$ & 1.49 \\
\hline $\begin{array}{l}\mathrm{D}_{\text {mean }} \\
\left(\times 10^{-3} \mathrm{~mm}^{2} / \mathrm{s}\right)\end{array}$ & $1.14 \pm 0.09$ & $1.63 \pm 0.1$ & 0.944 & 0.833 & $<0.001$ & 1.37 \\
\hline $\begin{array}{l}\mathrm{D} *_{\text {mean }} \\
\left(\times 10^{-3} \mathrm{~mm}^{2} / \mathrm{s}\right)\end{array}$ & $9.17 \pm 0.98$ & $8.94 \pm 0.13$ & 0.278 & 0.722 & 0.184 & 12.58 \\
\hline$f_{\text {mean }}(\%)$ & $28.58 \pm 1.37$ & $20.93 \pm 1.16$ & 0.5 & 0.944 & 0.007 & 29.83 \\
\hline
\end{tabular}

$$
\frac{S}{S_{0}}=\exp (-b \times \mathrm{ADC})
$$

$S$ : the SI with diffusion sensitization; $S_{0}$ : the SI without diffusion sensitization.

Briefly, for IVIM analysis, MATLAB (MathWorks, Natick, MA) was used to analyse the data measured on the diffusion-weighted images. The ADC was measured using a monoexponential model, and IVIM parameters were calculated using $8 b$ values fitted to a biexponential model (0-1200 $\mathrm{s} / \mathrm{mm}^{2}$ ) proposed by Le Bihan et al. [19].

$$
\frac{S}{S_{0}}=(1-f) x \exp ^{(-b D)}+f x \exp ^{(-b D *)}
$$

$S$ : the signal intensity in the pixel with diffusion gradient b; $S_{0}$ : the mean signal intensity in the pixel without diffusion gradient; $D\left(\times 10^{-3} \mathrm{~mm}^{2} / \mathrm{s}\right)$ : the true tissue diffusion coefficient (slow); $D *\left(\times 10^{-3} \mathrm{~mm}^{2} / \mathrm{s}\right)$ : the pseudo-perfusion (fast) diffusion coefficient; $f(\%)$ : the perfusion fractions reflecting the volume fraction of microcirculation.

For the region of interest (ROI), the areas with homogeneous signal intensities were selected; round areas (approximately 50-150 $\mathrm{mm}^{2}$ ) were drawn manually on the parametric maps with reference to the T2W images by 2 radiologists who had more than 5 years of MRI diagnosis experience. Notably, ROIs drawing should avoid the areas of focal SI changes, susceptibility artifacts, large necrosis and cystic areas and haemorrhage. All the data were measured 3 times to obtain mean values. T2WI and DCE-MR images were used to determine the lesion range on the IVIM parametric maps. All the data and volumes calculated were processed on an ADW 4.4 workstation (GE Healthcare). The changes in the IVIM parameters before NAC and after 2 cycles of NAC $\left(\Delta_{\mathrm{ADC}}, \Delta_{\mathrm{D}}\right.$, $\Delta_{\mathrm{D} *}$ and $\Delta_{f}$ ) were calculated with the same method of $\Delta_{\mathrm{V}}$.

2.5. Statistical Analysis. All the data were expressed as the mean \pm standard deviation. The normal distribution of all the data were tested with the Kolmogorov-Smirnov test. For $\mathrm{ADC}, \mathrm{D}, \mathrm{D} *$ and $f$, the differences before NAC and after 2 cycles of NAC were compared using Student's t-test.

Receiver operating characteristic (ROC) curves were used to evaluate the diagnostic performance of IVIM parameters to predict the response before and after NAC; the areas under the ROC curve (AUC), sensitivity, and specificity were also calculated. Diagnostic accuracy was defined according to AUC values (AUC $\geq 0.9$ indicated very good accuracy; $0.9>$
AUC $\geq 0.7$ indicated reasonable accuracy; $0.7>$ AUC $\geq 0.5$ indicated poor accuracy; $0.5>$ AUC indicated no accuracy). The optimal threshold (cutoff) was chosen according to the Youden index.

The relationship between the volume changes of the OS $\left(\Delta_{\mathrm{V}}\right)$ and the changes in the quantitative parameters $\left(\Delta_{\mathrm{ADC}}, \Delta_{\mathrm{D}}, \Delta_{\mathrm{D} *}\right.$ and $\left.\Delta_{f}\right)$ were determined using Spearman correlation analysis.

All the analyses were performed using SPSS 19.0 software for Windows (IBM Corporation, Armonk, NY, USA). P<0.05 was considered statistically significant.

The level of the intra-observer agreement was tested by Bland-Altman's variation coefficient using MedCalc (Version 18.2.1, Mariakerke, Belgium). The variation coefficient $<10 \%$ was defined as acceptable.

\section{Results}

3.1. Patients Therapeutic Response and the OS Volume before and after NAC. After 2 cycles of NAC, none patients were determined as CR and $\mathrm{PD}, 16$ patients were determined as $\mathrm{PR}$, and 2 patients were determined as stable disease, but the size decreased.

The volume of the OS after NAC decreased to varying degrees. The mean volume of the OS was $61.55 \pm 9.3 \mathrm{~cm}^{3}$ before NAC, and the mean volume of the OS was $40.26 \pm 4.1 \mathrm{~cm}^{3}$ after NAC $(\mathrm{P}<0.05)$.

3.2. MRI Imaging Findings and the Mean Values of ADC, D, $D *$ and $f$. The OS was heterogeneous, showing hypointensity on T1WI and hyperintensity on T2WI and DWI.

The intra-observer variation coefficients of the ADC, $\mathrm{D}, \mathrm{D} *$ and fvalue were low, with an average error of $5 \%$ coefficient of variance (less than 10\%), indicating a good intra-observer agreement level. After 2 cycles of NAC, some necrotic areas could be observed in the middle of the OS (Figure 1). After 2 cycles of NAC, the mean values of the ADC and $\mathrm{D}$ increased, and the $f$ values decreased significantly, while the $\mathrm{D} *$ values changed slightly $(\mathrm{P}<0.001,<0.001,=0.184$ and $<0.01$ respectively) (Figure 2 ; Table 1 ).

3.3. The Sensitivity and the Specificity of the $A D C, D, D *$ and $f$ Values. Among these parameters, the AUC values of ADC $(0.938)$ and $\mathrm{D}(0.943)$ were higher than those of $\mathrm{D} *(0.574)$ and $f(0.765)$. The sensitivity, specificity, and cu-toff values are listed in Table 1 (Figures 3 and 4). 


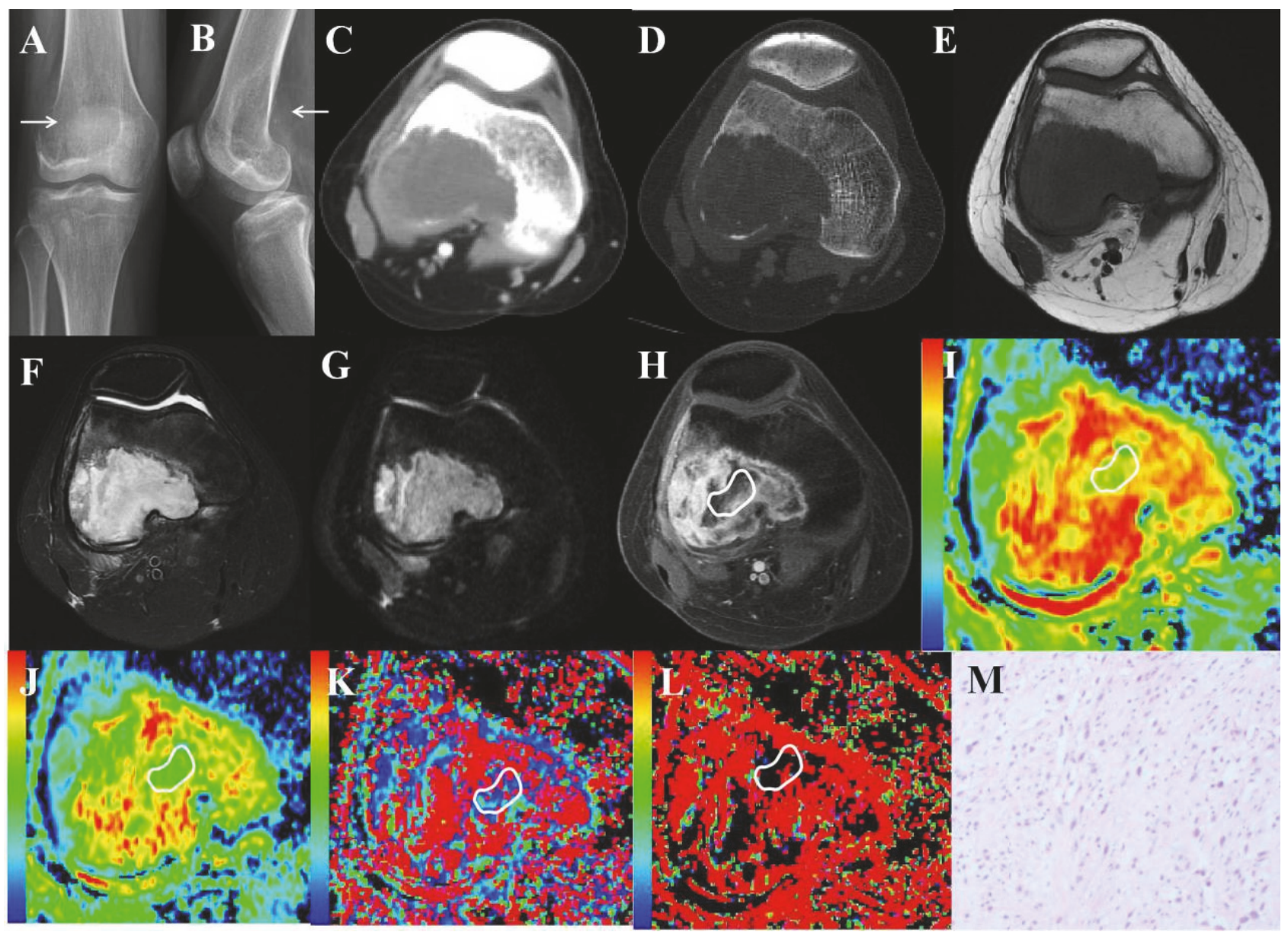

FIgURE 1: The medical images of a 19-year-old male patient with OS in the distal femoral metaphysis of the left knee. The bone destruction area located in the femoral metaphysis on X ray films (A, B) (arrows) and tomography imaging (CT) (C, D). The OS was hypointense on T1WI (E) and hyperintense on T2WI (F) and DWI (G). The margins of OS were ill-defined. The lesion enhanced after contrast injection (H). I-L: The pseudocolour maps of ADC, D, D* and $f$ derived from IVIM are displayed. M: HE staining $(\times 100)$. The necrotic areas were marked with curve (H, I, J, K).

3.4. The Correlation between $\Delta_{\mathrm{V}}$ and the IVIM Parameters. The mean $\Delta_{\mathrm{V}}$, the change in the IVIM parameters of the OS before and after NAC, was $19.32 \pm 2.93 \mathrm{~cm}^{3}$. The $\Delta_{\mathrm{ADC}}, \Delta_{\mathrm{D}}$, $\Delta_{\mathrm{D} *}$ and $\Delta_{f}$ values were $0.54 \pm 0.27 \times 10^{-3} \mathrm{~mm}^{2} / \mathrm{s}, 0.50 \pm 0.22$ $\times 10^{-3} \mathrm{~mm}^{2} / \mathrm{s}, 0.23 \pm 0.2 \times 10^{-3} \mathrm{~mm}^{2} / \mathrm{s}$ and $7.66 \pm 2.86 \%$. No significant correlations were found between the $\Delta_{\mathrm{V}}$ and $\Delta_{\mathrm{ADC}}, \Delta_{\mathrm{D}}, \Delta_{\mathrm{D} *}$ and $\Delta_{f}$ values $(\mathrm{P}>0.05)$.

\section{Discussion}

We investigated the value of IVIM in evaluating the osteosarcoma response to preoperative neoadjuvant chemotherapy in teens. We found that the ADC and D values increased significantly after 2 cycles of NAC, and the $\mathrm{D} *$ and $f$ values decreased; however, no significant difference was observed in $\mathrm{D} *$ before and after NAC. Furthermore, we found that the sensitivity and the specificity of the ADC and D were much higher than the $\mathrm{D} *$ and $f$ values. Nevertheless, no correlation was found between the volume and the IVIM parameter changes before and after NAC. All data suggested that IVIM could be a potential imaging biomarker of the OS chemotherapeutic response to preoperative NAC.

NAC before surgery and limb salvage treatment is the optimal and standard choice for osteosarcoma patients [31]. To preserve limb function, as well as to minimize the possible local recurrence and improve the quality of life, marginal resection was proposed for OS patients with favourable responses to NAC. In a study, with median follow-up of 5.5 years, the overall 5-year cumulative survival rate was $61.7 \%$, and excellent to good function of the affected limb was reported for $57.7 \%$ of OA patients undergoing marginal resection after NAC [32].

After NAC, functional MRI reflects the internal microenvironment changes in the tumour and predicts the response of the tumour to NAC $[33,34]$. IVIM differentiates microvascular perfusion activity (b values $<200 \mathrm{~s} / \mathrm{mm}^{2}$ ) from diffusion (b values $>200 \mathrm{~s} / \mathrm{mm}^{2}$ ). Therefore, IVIM was used widely in oncologic imaging and assessments of NAC [26, 27]. In our study, these results are consistent with the theory that cell density and microvessel density (MVD) might be altered 




FIGURE 2: The medical images of the same OS patient with Figure 1 after 2 cycles of NAC and femoral metaphysis surgery. The volume of the OS decreased compared with pre-NAC on CT imaging (A) (arrow). The OS was hypointense on T1WI with fat suppression (FS) (B) (arrow) and T1WI without FS (C). The lesion showed hyperintensity on T2WI (D) and DWI (E), and the margin of OS was relatively well-defined. The lesion showed rim-like enhancement after contrast injection (F-H), and the necrosis area was shown in OS (arrows). I-L: The pseudocolour maps of ADC, $\mathrm{D}, \mathrm{D} *$ and $f$ derived from IVIM were displayed. M: the OS specimen displayed, and the necrosis area was observed (arrows). $\mathrm{N}$ : HE staining $(\times 100)$. Fibrous tissue hyperplasia was observed in the lesion (white arrow). The residual degenerative tumour cells were also observed (black arrow).

greatly after NAC. After NAC, the true molecular diffusion and water molecules motion increased with less restriction due to larger extracellular spaces, yielding higher ADC and D values $[26,35]$. The $\mathrm{D}$ value was the marker of true molecular diffusion and water molecule diffusion related to the size of the intracellular and extracellular spaces; therefore, the larger the area of stromal tissue in the tumour appears, the higher the ADC and D values are [36]. After the course of NAC, cellular density in highly cellular tumour tissues decreased dramatically, leading to significant alterations in $\mathrm{ADC}$ and $\mathrm{D}$ values and better responses to NAC [27, 36]. These results were similar to those of previous studies [28, 37] and other tumours [38, 39]. These findings suggest that microcirculation or perfusion effects can differ from true tissue diffusion by multiple $b$ values and the biexponential curve fit analysis with IVIM imaging $[19,37]$. The ADC and $\mathrm{D}$ values had much higher AUC, sensitivity and specificity, consistent with previous studies [26, 28]. With the aid of IVIM, true molecular diffusion and water molecule motion in the capillary network could be evaluated with one-time imaging acquisition.

We also found that IVIM-derived $f$ values decreased and showed significant differences; however, they had lower AUC and sensitivity than the ADC and D values. Theoretically, the more the microvascular structures were destroyed and decreased in good responders to NAC, the greater the reduction in the $f$ value was. However, because of many factors, including the wide range of the $f$ values among the patients, poor interobserver agreement and T2 contributions of both perfusion and pure molecular diffusion compartments [27, 40], the $f$ values reported were different, and the $f$ usefulness was not identified [37]. In the biexponential IVIM model, $f$ is defined as the blood capillary and tumour tissue signal intensity ratio. All relaxation effects could be ignored if the relaxation times of the capillary blood and the tumour were similar. However, the T2 contributions of the tumour and blood capillaries were substantially different, and especially in tumours after NAC, T2 values tended to increase, which could lower the $f$ values artificially [40]. Therefore, the $f$ values may be useful in the evaluation of OS response to NAC; however, this should be done with much caution.

The $\mathrm{D} *$ value did not show significant changes before and after NAC and was not useful for the evaluation of tumour response. By comparison with $\mathrm{ADC}$ and $\mathrm{D}, \mathrm{D} *$ had its limitation, including less signal attenuation variability, high uncertainty, and poor measurement reproducibility [41, 


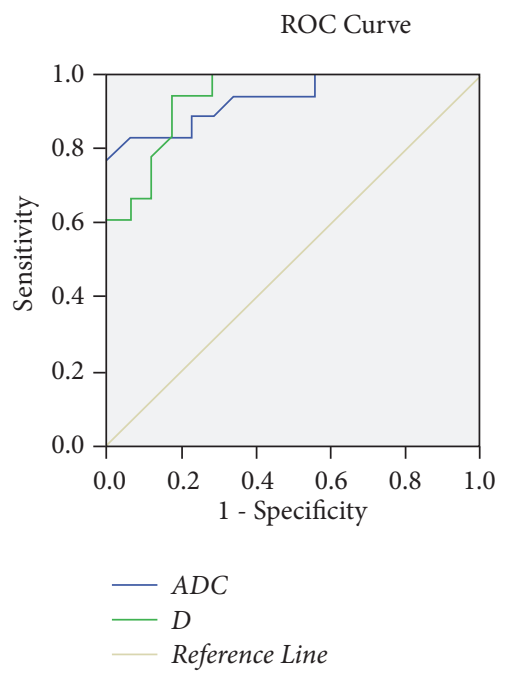

FIgUre 3: The ROC curve of the ADC and D values. The AUC values of ADC and D were 0.938 and 0.943 . The sensitivity and specificity of $\mathrm{ADC}$ and $\mathrm{D}$ values were $(0.778,1)$ vs $(0.944,0.833)$.



Figure 4: The ROC curve of the $\mathrm{D} *$ and $f$ values. The AUC values of $\mathrm{D} *$ and $f$ values were 0.574 and 0.765 . The sensitivity and specificity of $\mathrm{D} *$ and $f$ values were $(0.278,0.722)$ vs $(0.5,0.944)$.

42]. In OS before and after NAC, no significant differences were observed; we speculated that $\mathrm{D} *$ data might have large standard deviations that were unstable and dependent on the signal-to-noise ratio levels [15, 41]. In addition, the heterogeneity in tumour and noise variations could also affect the $\mathrm{D} *$ values [15]. Our findings are concordant with those of previous studies on cancers $[27,28,36]$, and the results suggested that $\mathrm{D} *$ has limited value in the assessment of tumour responses to CRT [41].

However, although the changes in the $\mathrm{V}, \mathrm{ADC}, \mathrm{D}$ and $f$ values before and after NAC showed significant differences, no correlation was found between $\Delta_{\mathrm{V}}$ and $\Delta_{\mathrm{ADC}}, \Delta_{\mathrm{D}}$ and $\Delta_{f}$. We believe this may be related to cellular degeneration, tissue necrosis, and individual differences [15, 22]. Further studies should be performed to obtain more accurate data.

Some limitations should be mentioned. First, the study size was relatively small, possibly having some effect on the results. Second, two NAC cycles for the observation are relative short; the majority OS patients were in the state of PR, so it lacks the investigation of the Long-term therapeutic efficacy. Third, we focused on the early therapeutic response, lacking long-term survival outcomes analysis. Fourth, no comparison was performed between the IVIMderived parameters and the dynamic contrast enhanced parameters, possibly reducing the convincing power of our data. We will continue to improve the study design regarding the above limitations in future studies.

In summary, $\mathrm{D}$ and $\mathrm{ADC}$ values are useful for evaluating and monitoring the efficacy of NAC in OS patients. Therefore, IVIM-MRI can be an effective potential imaging biomarker in early prediction of the therapeutic response to NAC in OS patients.

\section{Data Availability}

The quantitative data used to support the findings of this study are included within the article. The data are also available from the corresponding author upon request.

\section{Conflicts of Interest}

The author declares that they have no conflicts of interest.

\section{Acknowledgments}

This study was supported by a grant from the National Natural Science Foundation of China (no. 81701756) and from Sichuan Provincial Department of Education (no. 18ZB0215).

\section{References}

[1] R. L. Siegel, K. D. Miller, and A. Jemal, "Cancer statistics, 2018," CA: A Cancer Journal for Clinicians, vol. 68, no. 1, pp. 7-30, 2018.

[2] Z. Wu, J. Tan, X. Qin, B. Liu, and Z. Yuan, "Significance of circulating tumor cells in osteosarcoma patients treated by neoadjuvant chemotherapy and surgery," Cancer Management and Research, vol. Volume 10, pp. 3333-3339, 2018.

[3] K. P. Anfinsen, S. S. Devesa, F. Bray et al., "Age-periodcohort analysis of primary bone cancer incidence rates in the United States (1976-2005)," Cancer Epidemiology, Biomarkers \& Prevention, vol. 20, no. 8, pp. 1770-1777, 2011.

[4] S. Simpson, M. D. Dunning, S. de Brot, L. Grau-Roma, N. P. Mongan, and C. S. Rutland, "Comparative review of human and canine osteosarcoma: morphology, epidemiology, prognosis, treatment and genetics," Acta Veterinaria Scandinavica, vol. 59, no. 1, p. 71, 2017.

[5] Z. Nie and H. Peng, "Osteosarcoma in patients below 25 years of age: An observational study of incidence, metastasis, treatment and outcomes," Oncology Letters, vol. 16, no. 5, pp. 6502-6514, 2018. 
[6] H. Xu, X. Zhu, H. Bao et al., "Genetic and clonal dissection of osteosarcoma progression and lung metastasis," International Journal of Cancer, vol. 143, no. 5, pp. 1134-1142, 2018.

[7] S. P. Zhang, X. Li, H. Li, X. H. Sun, and X. F. Yan, "Significance of neoadjuvant chemotherapy (NACT) in limb salvage treatment of osteosarcoma and its effect on GLS1 expression," European Review for Medical and Pharmacological Sciences, vol. 22, no. 19, pp. 6538-6544, 2018.

[8] Z. Huang and C. Lou, "Application of the alteration uptake ratio of $99 \mathrm{mTc}-\mathrm{MIBI}$ scintigraphy for evaluating the efficacy of neoadjuvant chemotherapy in osteosarcoma patients," Hellenic Journal of Nuclear Medicine, vol. 21, no. 1, pp. 55-59, 2018.

[9] D. J. Harrison, D. S. Geller, J. D. Gill, V. O. Lewis, and R. Gorlick, "Current and future therapeutic approaches for osteosarcoma," Expert Review of Anticancer Therapy, vol. 18, no. 1, pp. 39-50, 2018.

[10] A. Luetke, P. A. Meyers, I. Lewis, and H. Juergens, "Osteosarcoma treatment-where do we stand? A state of the art review," Cancer Treatment Reviews, vol. 40, no. 4, pp. 523-532, 2014.

[11] J. K. Anninga, H. Gelderblom, M. Fiocco et al., "Chemotherapeutic adjuvant treatment for osteosarcoma: where do we stand?" European Journal of Cancer, vol. 47, no. 16, pp. 24312445, 2011.

[12] M. Collins, M. Wilhelm, R. Conyers et al., "Benefits and adverse events in younger versus older patients receiving neoadjuvant chemotherapy for osteosarcoma: findings from a metaanalysis," Journal of Clinical Oncology, vol. 31, no. 18, pp. 23032312, 2013.

[13] M. P. Van Der Paardt, M. B. Zagers, R. G. H. Beets-Tan, J. Stoker, and S. Bipat, "Patients who undergo preoperative chemoradiotherapy for locally advanced rectal cancer restaged by using diagnostic MR imaging: A systematic review and metaanalysis," Radiology, vol. 269, no. 1, pp. 101-112, 2013.

[14] R. G. H. Beets-Tan, G. L. Beets, R. F. A. Vliegen et al., "Accuracy of magnetic resonance imaging in prediction of tumour-free resection margin in rectal cancer surgery," The Lancet, vol. 357, no. 9255, pp. 497-504, 2001.

[15] D.-M. Koh, D. J. Collins, and M. R. Orton, "Intravoxel incoherent motion in body diffusion-weighted MRI: Reality and challenges," American Journal of Roentgenology, vol. 196, no. 6, pp. 1351-1361, 2011.

[16] L. Curvo-Semedo, D. M. J. Lambregts, M. Maas et al., "Rectal cancer: assessment of complete response to preoperative combined radiation therapy with chemotherapy-conventional MR volumetry versus diffusion-weighted MR imaging," Radiology, vol. 260, no. 3, pp. 734-743, 2011.

[17] M. Iima and D. Le Bihan, "Clinical intravoxel incoherent motion and diffusion MR imaging: Past, present, and future," Radiology, vol. 278, no. 1, pp. 13-32, 2016.

[18] C. Federau, "Intravoxel incoherent motion MRI as a means to measure in vivo perfusion: A review of the evidence," NMR in Biomedicine, vol. 30, no. 11, Article ID e3780, 2017.

[19] D. Le Bihan, E. Breton, D. Lallemand, M.-L. Aubin, J. Vignaud, and M. Laval-Jeantet, "Separation of diffusion and perfusion in intravoxel incoherent motion MR imaging," Radiology, vol. 168, no. 2, pp. 497-505, 1988.

[20] T. Bäuerle, L. Seyler, M. Münter et al., "Diffusion-weighted imaging in rectal carcinoma patients without and after chemoradiotherapy: A comparative study with histology," European Journal of Radiology, vol. 82, no. 3, pp. 444-452, 2013.

[21] D. J. Le Bihan, "Differentiation of benign versus pathologic compression fractures with diffusion-weighted MR imaging: A closer step toward the 'Holy Grail' of tissue characterization?" Radiology, vol. 207, no. 2, pp. 305-307, 1998.

[22] J. Wang, M. Sun, D. Liu et al., "Correlation between apparent diffusion coefficient and histopathology subtypes of osteosarcoma after neoadjuvant chemotherapy," Acta Radiologica, vol. 58, no. 8, pp. 971-976, 2017.

[23] O. Jalnefjord, M. Andersson, M. Montelius et al., "Comparison of methods for estimation of the intravoxel incoherent motion (IVIM) diffusion coefficient (D) and perfusion fraction (f)," Magnetic Resonance Materials in Physics, Biology and Medicine, vol. 31, no. 6, pp. 715-723, 2018.

[24] H. D. Zuo and X. M. Zhang, "Could intravoxel incoherent motion diffusion-weighted magnetic resonance imaging be feasible and beneficial to the evaluation of gastrointestinal tumors histopathology and the therapeutic response?" World Journal of Radiology, vol. 10, no. 10, pp. 116-123, 2018.

[25] H.-J. Lee, S. Y. Rha, Y. E. Chung et al., “Tumor perfusion-related parameter of diffusion-weighted magnetic resonance imaging: Correlation with histological microvessel density," Magnetic Resonance in Medicine, vol. 71, no. 4, pp. 1554-1558, 2014.

[26] Q. Xu, Y. Xu, H. Sun et al., "Quantitative intravoxel incoherent motion parameters derived from whole-tumor volume for assessing pathological complete response to neoadjuvant chemotherapy in locally advanced rectal cancer," Journal of Magnetic Resonance Imaging, vol. 48, no. 1, pp. 248-258, 2018.

[27] Y. Kim, S. H. Kim, H. W. Lee et al., "Intravoxel incoherent motion diffusion-weighted MRI for predicting response to neoadjuvant chemotherapy in breast cancer," Magnetic Resonance Imaging, vol. 48, pp. 27-33, 2018.

[28] Y.-C. Wang, D.-Y. Hu, X.-M. Hu et al., "Assessing the early response of advanced cervical cancer to neoadjuvant chemotherapy using intravoxel incoherent motion diffusionweighted magnetic resonance imaging: A pilot study," Chinese Medical Journal, vol. 129, no. 6, pp. 665-671, 2016.

[29] E. A. Eisenhauer, P. Therasse, J. Bogaerts et al., "New response evaluation criteria in solid tumours: revised RECIST guideline (version 1.1)," European Journal of Cancer, vol. 45, no. 2, pp. 228 247, 2009.

[30] H. D. Zuo and W. W. Yao, "The role and the potential regulatory pathways of high expression of forkhead box $\mathrm{Cl}$ in promoting tumor growth and metastasis of basal-like breast cancer," Journal of B.U.ON., vol. 21, no. 4, pp. 818-825, 2016.

[31] Y. Yang, L. Han, Z. He et al., "Advances in limb salvage treatment of osteosarcoma," Journal of Bone Oncology, vol. 10, pp. 36-40, 2018.

[32] M. Xu, S. Xu, and X. Yu, "Marginal resection for osteosarcoma with effective neoadjuvant chemotherapy: long-term outcomes," World Journal of Surgical Oncology, vol. 12, no. 1, p. 341,2014

[33] F. He, L. Qin, Q. Bao et al., "Pre-Operative chemotherapy response assessed by contrast-enhanced MRI can predict the prognosis of Enneking surgical margins in patients with osteosarcoma," Journal of Orthopaedic Research, 2018.

[34] J. Bajpai, S. Gamnagatti, R. Kumar et al., "Role of MRI in osteosarcoma for evaluation and prediction of chemotherapy response: correlation with histological necrosis," Pediatric Radiology, vol. 41, no. 4, pp. 441-450, 2011.

[35] H. Zuo and Q. Yang, "The potential pathway of FOXC1 high expression in regulating the proliferation, migration, cell cycle and epithelial-mesenchymal transition of basal-like breast cancer and in vivo imaging," Journal of B.U.ON., vol. 23, no. 3, pp. 720-728, 2018. 
[36] H.-B. Zhu, X.-Y. Zhang, X.-H. Zhou et al., "Assessment of pathological complete response to preoperative chemoradiotherapy by means of multiple mathematical models of diffusionweighted MRI in locally advanced rectal cancer: A prospective single-center study," Journal of Magnetic Resonance Imaging, vol. 46, no. 1, pp. 175-183, 2017.

[37] S. Nougaret, H. A. Vargas, Y. Lakhman et al., "Intravoxel incoherent motion-derived histogram metrics for assessment of response after combined chemotherapy and radiation therapy in rectal cancer: initial experience and comparison between single-section and volumetric analyses," Radiology, vol. 280, no. 2, pp. 446-454, 2016.

[38] S. Woo, J. M. Lee, J. H. Yoon, I. Joo, J. K. Han, and B. I. Choi, "Intravoxel incoherent motion diffusion-weighted $\mathrm{mr}$ imaging of hepatocellular carcinoma: Correlation with enhancement degree and histologic grade," Radiology, vol. 270, no. 3, pp. 758767, 2014.

[39] Y. Xiao, J. Pan, Y. Chen, Y. Chen, Z. He, and X. Zheng, "Intravoxel incoherent motion-magnetic resonance imaging as an early predictor of treatment response to neoadjuvant chemotherapy in locoregionally advanced nasopharyngeal carcinoma," Medicine, vol. 94, no. 24, p. e973, 2015.

[40] A. Lemke, F. B. Laun, D. Simon, B. Stieltjes, and L. R. Schad, "An in vivo verification of the intravoxel incoherent motion effect in diffusion-weighted imaging of the abdomen," Magnetic Resonance in Medicine, vol. 64, no. 6, pp. 1580-1585, 2010.

[41] A. Andreou, D. M. Koh, D. J. Collins et al., "Measurement reproducibility of perfusion fraction and pseudodiffusion coefficient derived by intravoxel incoherent motion diffusion-weighted MR imaging in normal liver and metastases," European Radiology, vol. 23, no. 2, pp. 428-434, 2013.

[42] S. Che, X. Zhao, Y. Ou et al., "Role of the intravoxel incoherent motion diffusion weighted imaging in the pre-treatment prediction and early response monitoring to neoadjuvant chemotherapy in locally advanced breast cancer," Medicine (United States), vol. 95, no. 4, p. e2420, 2016. 




\section{Enfincering}


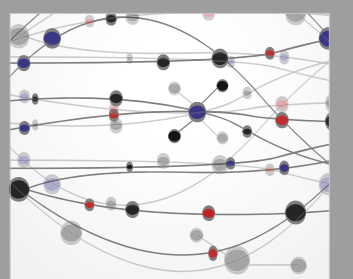

\section{Rotating \\ Machinery}

The Scientific World Journal



Submit your manuscripts at

www.hindawi.com
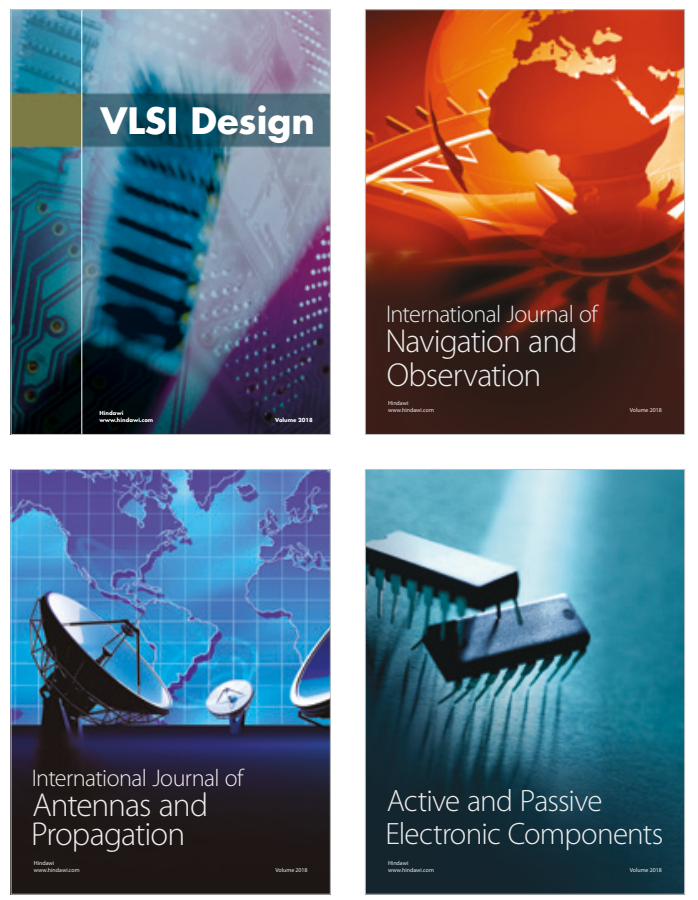


\section{Advances \\ Multimedia}
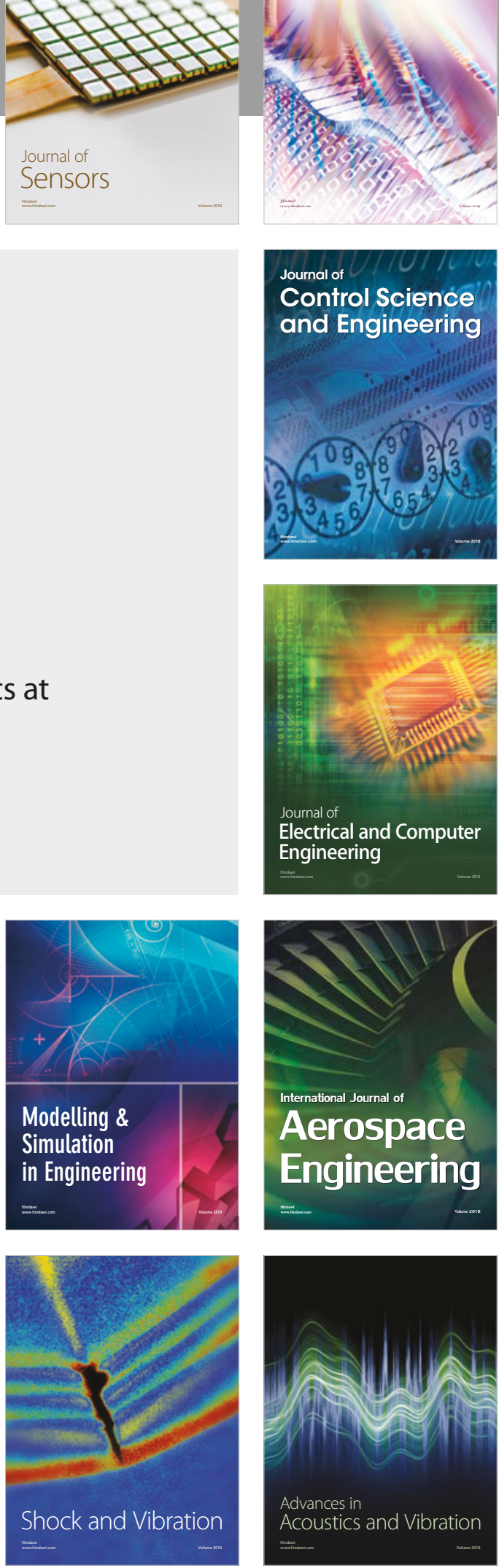